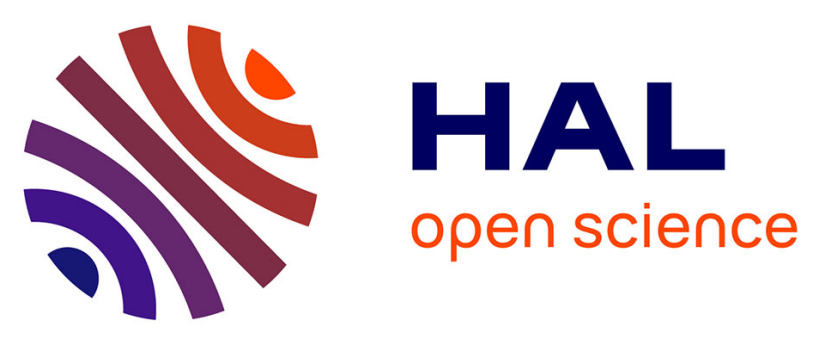

\title{
Distribution discontinuities detection using algebraic technique for spectrum sensing in cognitive radio networks
}

\author{
Guibène Wael, Hayar Aawatif, Turki Monia
}

\section{- To cite this version:}

Guibène Wael, Hayar Aawatif, Turki Monia. Distribution discontinuities detection using algebraic technique for spectrum sensing in cognitive radio networks. CrownCom 2010,, 5th International Conference on Cognitive Radio Oriented Wireless Networks and Communications, Jun 2010, Cannes, France. pp.1 - 5, 10.4108/ICST.CROWNCOM2010.9183 . hal-00689674

\section{HAL Id: hal-00689674 https://hal.science/hal-00689674}

Submitted on 20 Apr 2012

HAL is a multi-disciplinary open access archive for the deposit and dissemination of scientific research documents, whether they are published or not. The documents may come from teaching and research institutions in France or abroad, or from public or private research centers.
L'archive ouverte pluridisciplinaire HAL, est destinée au dépôt et à la diffusion de documents scientifiques de niveau recherche, publiés ou non, émanant des établissements d'enseignement et de recherche français ou étrangers, des laboratoires publics ou privés. 


\section{Distribution Discontinuities Detection using Algebraic Technique for Spectrum Sensing in Cognitive Radio}

\author{
Wael Guibène and Aawatif Hayar \\ EURECOM - Mobile Communications Dept. \\ P.O. Box 193, 06904 Sophia Antipolis, France \\ Email: Wael.Guibenedeurecom.fr \\ Email: Aawatif.Hayar@eurecom.fr
}

\author{
Monia Turki \\ ENIT - Unité Signaux et Systèmes. \\ BP 37, Le Belvedere, 1002 Tunis, Tunisia \\ Email: m.turki@enit.rnu.tn
}

\begin{abstract}
In this paper ${ }^{1}$, we propose a new Spectrum Sensing technique for spectrum distribution discontinuities detection using algebraic detection (AD). The presented mathematical background leading to such technique is based on operational calculus and differential algebra which offers a new stand point in sensing theory. Even if this background is quiet heavy, the sensing algorithm related to this technique is nevertheless very simple and remains with extremely low complexity, comparable even to the Energy Detection technique (ED). Simulations results show good performance of the proposed technique in terms of reliability and accuracy with low complexity and good robustness in noisy context.
\end{abstract}

Index Terms-Cognitive radio, sensing algorithm, energy detector, distribution discontinuities, algebraic detector, change point detection.

\section{INTRODUCTION}

Recently, wireless technologies have known a new era of expanse and development. Different services are available with high quality of service (QoS). Formally, in wireless networks, implementing new services and deploying new users means assigning and occupying new frequency band. Today and due to this rise of technologies, the main resource on which are based the wireless communications -i.e spectrum- is becoming scarce and misused. For instance measurement lead by the FCC (Federal Communication Commission) in the USA have shown that in some regions and/or at some day intervals up to 70 percent of the statically allocated spectrum is left idle [1]. Facing this misuse, the FCC recommended deploying unlicensed users in the wireless networks. These unlicensed users also called secondary users (SUs) are allowed to use those idle wireless resources only when the licensed users, also called primary user (PU), is not using them so they do not interfere with its transmissions.

In order to make such a concept of spectrum sharing feasible, SU are cognitive radios (CRs) deployed in the primary

\footnotetext{
${ }^{1}$ For the first author, the research leading to these results has received funding from the European Community's Seventh Framework Programme (FP7/2007-2013) under grant agreement SACRA $n^{\circ} 249060$.
}

networks. CR as introduced by Joseph Mitola [2] is a self aware and intelligent device that can adapt itself to the wireless environment changes by firstly detecting them, then adapting its radio parameters to the new opportunities.

Spectrum Sensing in CR aims at finding the holes in the PU transmission which are the best opportunities, in terms of interference, to be used by the SU. Many statistical approaches already exist. The easiest to implement and the reference one in terms of complexity is still the energy detector (ED) [4]. Nevertheless, the ED is highly sensitive to noise and does not perform well in low signal to noise ratio (SNR). Other advanced techniques based on signals modulations and exploiting some of the transmitted signals inner features were also developed [3]. For instance, the cyclostationary features detector (CFD) exploits the built-in cyclic properties of the PU received signal. The CFD do have a great robustness to noise compared to ED but its high complexity is still a consequent drawback. Other techniques that were developed by Eurécom Institute based on the model selection and entropy [7] [5] [6]. The main idea consists in finding a candidate that best fits the statistics of the observed.

In this paper, we propose a different standpoint for spectrum sensing. The algebraic approaches were originally developed for locating spikes, which are neuronal activities, in electroencephalograms (EEGs). We suggested in this work to apply these spike detection spectrum distribution discontinuities in the context of spectrum sensing for CR.

The first step was to model the spectrum as a piecewise polynomial, then by using some mathematical framework inspired from [9], the sensing problem is then casted as a change point detection in the spectrum of the PU transmission as detailed in [8]. Despite the heaviness of the mathematical framework, the derived sensing algorithm is still easy to implement as it is a simple filtering of the noisy spectrum.

The paper is arranged as follows: Section II introduces the model used to describe the proposed sensing algorithm. Then, the AD implementation is presented in Section III. In Section IV, the performance evaluation and advantages are described, and a comparison with some statistical approaches 
is given. Finally, Section V concludes about the presented work in this paper.

\section{SySTEM MODEL}

In this section we investigate the system model considered through this paper. In this system, the received signal at time $n$, denoted by $y_{n}$, can be modeled as:

$$
y_{n}=A_{n} s_{n}+e_{n}
$$

where $A_{n}$ being the transmission channel gain, $s_{n}$ is the transmit signal sent from primary user and $e_{n}$ is an additive corrupting noise.

In order to avoid interferences with the primary (licensed) system, the cognitive radio needs to sense its radio environment whenever it wants to access available spectrum resources. The goal of spectrum sensing is to decide between two conventional hypotheses modeling the spectrum occupancy:

$$
y_{n}= \begin{cases}e_{n} & \mathrm{H}_{0} \\ A_{n} s_{n}+e_{n} & \mathrm{H}_{1}\end{cases}
$$

The sensed sub-band is assumed to be a white area if it contains only a noise component, as defined in $\mathrm{H}_{0}$; while, once there exist primary user signals drowned in noise in a specific band, as defined in $\mathrm{H}_{1}$, we infer that the band is occupied. The key parameters of all spectrum sensing algorithms are the false alarm probability $P_{F}$ and the detection probability $P_{D}$. $P_{F}$ is the probability that the sensed sub-band is classified as a PU data while actually it contains noise, thus $P_{F}$ should be kept as small as possible.

$P_{D}$ is the probability of classifying the sensed sub-band as a PU data when it is truly present, thus sensing algorithm tend to maximize $P_{D}$. To design the optimal detector on NeymanPearson criterion, we aim on maximizing the overall $P_{D}$ under a given overall $P_{F}$.

According to those definitions, the probability of false alarm is given by:

$$
P_{F}=P\left(\mathrm{H}_{1} \mid \mathrm{H}_{0}\right)=P\left(y_{n} \text { is present } \mid \mathrm{H}_{0}\right)
$$

that is the probability of the spectrum detector having detected a signal given the hypothesis $H_{0}$, and $P_{D}$ the probability of detection is expressed as:

$$
\begin{aligned}
P_{D} & =1-P_{M}=1-P\left(\mathrm{H}_{0} \mid \mathrm{H}_{1}\right) \\
& =1-P\left(y_{n} \text { is absent } \mid \mathrm{H}_{1}\right)
\end{aligned}
$$

which represents the probability of the detector having detected a signal under hypothesis $H_{1}$, where $P_{M}$ indicates the probability of missed detection.

In order to infer on the nature of the received signal, we calculate a threshold for each of the detectors via a Monte Carlo simulation. The decision threshold is determined using the required probability of false alarm $P_{F}$ given by (3). The threshold $T h$ for a given false alarm probability is determined by solving the equation:

$$
P_{F}=P\left(y_{n} \text { is present } \mid \mathrm{H}_{0}\right)=1-F_{H_{0}}(T h)
$$

where $F_{H_{0}}$ denote the cumulative distribution function (CDF) under $H_{0}$.

\section{Algebraic Detection Technique}

\section{A. Mathematical Background}

In this Section we give an overview of the mathematical background leading to the algebraic detection technique.

The input signal is the amplitude spectrum of the received noisy signal. We assume that its mathematical representation is a piecewise regular signal:

$$
Y(f)=\sum_{i=1}^{K} \chi_{i}\left[f_{i-1}, f_{i}\right](f) p_{i}\left(f-f_{i-1}\right)+n(f)
$$

where: $\chi_{i}\left[f_{i-1}, f_{i}\right]$ : the characteristic function of the interval $\left[f_{i-1}, f_{i}\right],\left(p_{i}\right)_{i \in[1, K]}$ : an $N^{t h}$ order polynomials series, $\left(f_{i}\right)_{i \in[1, K]}$ : the discontinuity points resulting from multiplying each $p_{i}$ by a $\chi_{i}$ and $n(f)$ :the additive corrupting noise.

Now, let $X(f)$ the clean version of the received signal given by:

$$
X(f)=\Sigma_{i=1}^{K} \chi_{i}\left[f_{i-1}, f_{i}\right](f) p_{i}\left(f-f_{i-1}\right)
$$

And let $b$, the frequency band, given such as in each interval $I_{b}=\left[f_{i-1}, f_{i}\right]=[\nu, \nu+b], \nu \geq 0$ one and only one change point occurs in the interval $I_{b}$.

Now denoting $X_{\nu}(f)=X(f+\nu), f \in[0, b]$ for the restriction of the signal in the interval $I_{b}$ and redefine the change point relatively to $I_{b}$ say $f_{\nu}$ given by:

$\left\{f_{\nu}=0\right.$ if $X_{\nu}$ is continuous

$\left\{<f_{\nu} \leq b\right.$ otherwise

Now the $N^{t h}$ derivative of $X_{\nu}(f)$ in the sense of Distributions Theory is:

$$
\frac{d^{N}}{d f^{N}} X_{\nu}(f)=\left[X_{\nu}(f)\right]^{(N)}+\sum_{k=1}^{N} \mu_{N-k} \delta\left(f-f_{\nu}\right)^{(k-1)}
$$

where: $\mu_{k}$ is the jump of the $k^{t h}$ order derivative at the unique assumed change point: $f_{\nu}$

$$
\mu_{k}=X_{\nu}^{(k)}\left(f_{\nu}^{+}\right)-X_{\nu}^{(k)}\left(f_{\nu}^{-}\right)
$$

with : $\left\{\begin{array}{l}\left.\mu_{k}=0\right\rfloor_{k=1 . . N} \text { if there is no change point. } \\ \left.\mu_{k} \neq 0\right\rfloor_{k=1 . . N} \text { if the change point is in } I_{b} .\end{array}\right.$

$\left[\mathrm{X}_{\nu}(f)\right]^{(N)}$ is the regular derivative part of the $N^{t h}$ derivative of the signal.

The spectrum sensing problem is now casted as a change point $f_{\nu}$ detection problem. Several estimators can be derived from the equation 8 . For example any derivative order $\mathrm{N}$ can be taken and depending on this order the equation is solved in the operational domain and back to frequency domain the estimator is deduced.

In a matter of reducing the complexity of the frequency direct resolution, the equation 8 is transposed to the operational domain, using the Laplace transform:

$$
\begin{aligned}
L\left(X_{\nu}(f)^{(N)}\right) & \left.=s^{N} \widehat{X_{\nu}}(s)-\sum_{m=0}^{N-1} s^{N-m-1} \frac{d^{m}}{d f^{m}} X_{\nu}(f)\right\rfloor_{f=0} \\
& =e^{-s f_{\nu}}\left(\mu_{N-1}+s \mu_{N-2}+. .+s^{N-1} \mu_{0}\right)
\end{aligned}
$$


Given the fact the initial conditions and the jump of the derivatives of $X_{\nu}(f)$ are unknown parameters to the problem, in a first time we are going to annihilate the jump values $\mu_{0}, \mu_{1}, \ldots, \mu_{N-1}$ then the initial conditions. After some calculations steps detailed, we finally obtain:

$$
\sum_{k=0}^{N-1}\left(\begin{array}{l}
N \\
k
\end{array}\right) \cdot f_{\nu}^{N-k} \cdot\left(s^{N} \widehat{X_{\nu}}(s)\right)^{(N+k)}=0
$$

In the actual context, the noisy observation of the amplitude spectrum $Y(f)$ is taken instead of $X_{\nu}(f)$. As taking derivative in the operational domain is equivalent to high-pass filtering in frequency domain, which may help amplifying the noise effect. It is suggested to divide the whole equation 11 by $s^{l}$ which in the frequency domain will be equivalent to an integration if $l>2 N$, we thus obtain:

$$
\sum_{k=0}^{N-1}\left(\begin{array}{l}
N \\
k
\end{array}\right) \cdot f_{\nu}^{N-k} \cdot \frac{\left(s^{N} \widehat{X}_{\nu}(s)\right)^{(N+k)}}{s^{l}}=0
$$

Since there is no unknown variables anymore, the equation 12 is now transformed back to the frequency domain, we obtain the polynomial to be solved on each sensed sub-band:

$$
\sum_{k=0}^{N-1}\left(\begin{array}{l}
N \\
k
\end{array}\right) \cdot f_{\nu}^{N-k} \cdot L^{-1}\left[\frac{\left(s^{N} \widehat{X_{\nu}}(s)\right)^{(N+k)}}{s^{l}}\right]=0
$$

And denoting:

$\varphi_{k+1}=L^{-1}\left[\frac{\left(s^{N} \widehat{X}_{\nu}(s)\right)^{(N+k)}}{s^{l}}\right]=\int_{0}^{+\infty} h_{k+1}(f) \cdot X(\nu-f) \cdot d f$

where: $h_{k+1}(f)=\left\{\begin{array}{l}\frac{\left(f^{l}(b-f)^{N+k}\right)^{(k)}}{(l-1) !}, 0<f<b \\ 0, \text { otherwise }\end{array}\right.$

To summarize, we have shown that on each interval $[0, b]$, for the noise-free observation the change points are located at frequencies solving:

$$
\sum_{k=0}^{N}\left(\begin{array}{l}
N \\
k
\end{array}\right) \cdot f_{\nu}^{N-k} \cdot \varphi_{k+1}=0
$$

\section{B. Implementation Issues}

The proposed algorithm is implemented as a filter bank which is composed of $N$ filters mounted in a parallel way. The impulse response of each filter is: $\mathrm{h}_{k+1}(f)=$ $\left\{\begin{array}{l}\frac{\left(f^{l}(b-f)^{N+k}\right)^{(k)}}{(l-1) !}, 0<f<b \\ 0, \text { otherwise }\end{array} \quad\right.$ where $k \in[0 . . N-1]$ and $l$ is chosen such as $l>2 \times N$. The proposed expression of $\left.h_{k+1}\right\rfloor_{k \in[0 . . N-1]}$ was determined by modeling the spectrum by a piecewise regular signal in frequency domain and casting the problem of spectrum sensing as a change point detection in the primary user transmission [8]. Finally, in each stage of the filter bank, we compute the following equation:

$$
\varphi_{k+1}(f)=\int_{0}^{+\infty} h_{k+1}(\nu) \cdot X(f-\nu) \cdot d \nu
$$

Equation (15) is then used to detect spectrum discontinuities and to find the intervals of interest.

\section{Algorithm Disctrete Implementaton}

The proposed algorithm in its discrete implementation is a filter bank composed of $N$ filters mounted in a parallel way. The impulse response of each filter is:

$$
h_{k+1, n}=\left\{\begin{array}{l}
\frac{\left(n^{l}(b-n)^{N+k}\right)^{(k)}}{(l-1) !}, 0<n<b \\
0, \text { otherwise }
\end{array}\right.
$$

where $k \in[0 . . N-1]$ and $l$ is chosen such as $l>2 \times N$. The proposed expression of $\left.h_{k+1, n}\right\rfloor_{k \in[0 . . N-1]}$ was determined by modeling the spectrum by a piecewise regular signal in frequency domain and casting the problem of spectrum sensing as a change point detection in the primary user transmission [8]. Finally, in each detected interval $\left[n_{\nu_{i}}, n_{\nu_{i+1}}\right]$, we compute the following equation:

$$
\varphi_{k+1}=\sum_{m=n_{\nu_{i}}}^{n_{\nu_{i+1}}} W_{m} h_{k+1, m} X_{m}
$$

where $W_{m}$ are the weights for numeric integration defined by:

$$
\begin{array}{ll}
W_{0}=W_{M}=0.5 & \\
W_{m}=1 & \text { otherwise }
\end{array}
$$

In order to infer whether the primary user is present in the detected intervals, a decision function is computed as following:

$$
D f=\left\|\prod_{k=0}^{N} \varphi_{k+1}\left(n_{\nu}\right)\right\|
$$

After the detection of spectrum discontinuities by solving equation (15), the decision is then made by computing the threshold Th to the mean value over the detected intervals.

\section{Performance Evaluation}

First of all, let's give some key notes on the ED. ED is the most common method for spectrum sensing because of its noncoherency and low complexity. The energy detector measures the received energy during a finite time interval and compares it to a predetermined threshold. That is, the test statistic of the energy detector is:

$$
\sum_{n=1}^{M}\left\|y_{n}\right\|^{2}
$$

where $M$ is the number of samples of the received signal $x_{n}$.

Traditional ED can be simply implemented as a spectrum analyzer. A threshold used for primary user detection is highly susceptible to unknown or changing noise levels. Even if the threshold would be set adaptively, presence of any in-band interference would confuse the energy detector.

Since the complexity of sensing algorithms is a major concern in implementation. As ED is well known for its simplicity, 
the comparison is made with reference to it. Denoting $M$ the number of samples of the received signal $y_{n}$ and $N$ is the model order of the AD, we show that the AD complexity is $N M$ and the ED complexity is $M$. From these results, we clearly see that the proposed sensing algorithm has a comparable complexity level as the energy detector. Table I summarizes the complexity of the two techniques.

\begin{tabular}{|l|c|}
\hline Sensing technique & Complexity \\
\hline Energy detector & $\mathrm{M}$ \\
Algebraic detector & $\mathrm{NM}$ \\
\hline
\end{tabular}

TABLE I

COMPLEXITY COMPARISON OF THE DIFFERENT SENSING TECHNIQUES

For simulation results, the choice of the DVB-T primary user system is justified by the fact that most of the primary user systems utilize the OFDM modulation format. The considered model is an Additive White Gaussian Noise (AWGN) channel. The simulation scenarios are generated by using different combinations of parameters given in Table II.

\begin{tabular}{|l|l|}
\hline Bandwidth & $8 \mathrm{MHz}$ \\
\hline Mode & $2 \mathrm{~K}$ \\
\hline Guard interval & $1 / 4$ \\
\hline Frequency-flat & Single path \\
\hline Sensing time & $1.25 \mathrm{~ms}$ \\
\hline Location variability & $10 \mathrm{~dB}$ \\
\hline
\end{tabular}

TABLE II

THE TRANSMITTED DVB-T PRIMARY USER SIGNAL PARAMETERS

Figure 1 shows the detected change points by the algebraic technique where: the blue signal is the simulated OFDM signal and the green stars are the detected change points.

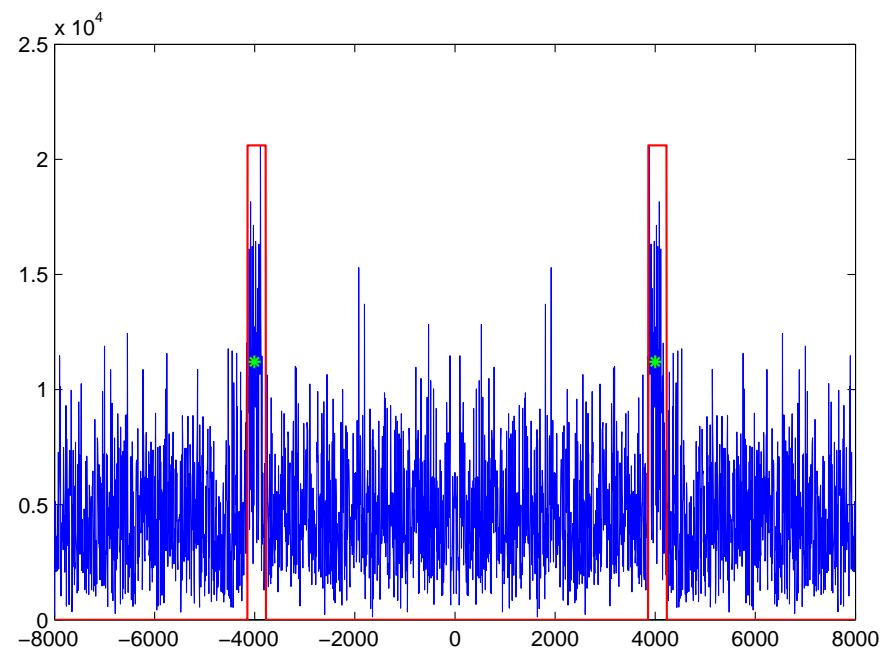

Fig. 1. Change point detection with $\mathrm{SNR}=-8 \mathrm{~dB}$.

Fig. ?? reports the comparison in terms of Probability of Detection Vs. SNR between the Energy Detector $(E D)$ and the two first Algebraic Detectors: $\left(A D_{2}\right)$ and $\left(A D_{3}\right)$, for $P_{F}=0.05$ and $\mathrm{SNR}$ ranging in -40 to $0 \mathrm{dBs}$.
The threshold level for each detector is computed with function of the probability of false alarm $P_{F}$ with respect to Equation 5.

This figure clearly shows that the proposed sensing algorithm is quite robust to noise. These curves show also that the detection rate goes higher as the polynomial order gets higher.

This result is to be expected as the higher the polynomial order is, the more accurate the approximation a polynomial is. Nevertheless, it is to be noticed that this gain in precision is implies a higher complexity in the algorithms implementation.

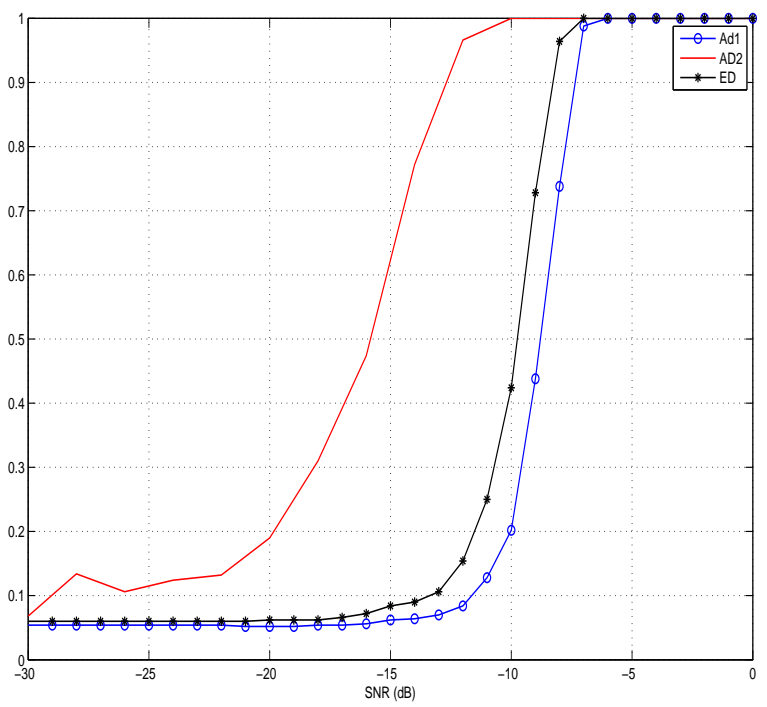

Fig. 2. Probability of detection vs. SNR for the three detectors: energy detector $(E D), 1^{\text {st }}$ order algebraic detector $\left(A D_{1}\right)$ and $2^{\text {nd }}$ order algebraic detector $\left(A D_{3}\right)$ with $P_{F}=0.05$.

In Figure 3, we plot the ROC curve at an $\mathrm{SNR}=-15 \mathrm{~dB}$. We clearly see that for the proposed technique that the higher the order is, the more performing the detector gets.

\section{CONCLUSION}

In this paper, we presented a new approach for spectrum sensing based on the detection of spectrum distribution discontinuities using algebraic method. Despite the heaviness of the mathematical background, the implementation of such a technique is easy and for low orders is comparable to the reference technique. The proposed algorithm takes in consideration the noise effect in its inner structure which gives good, accurate and reliable results even in very low SNRs.

\section{REFERENCES}

[1] FCC, "Spectrum Policy Task Force Report, ET Docket No. 02-155, Nov. 2002 "

[2] J. Mitola, "Cognitive radio: An integrated agent architecture for software defined radio", Doctor of Technology, Royal Inst. Technol. (KTH), Stockholm, Sweden, 2000.

[3] A. Sahai and D. Cabric, "Spectrum sensing: fundamental limits and practical challenges", in Proc. IEEE International Symposium on New Frontiers in Dynamic Spectrum Access Networks (DySPAN), (Baltimore, MD), Nov. 2005. 


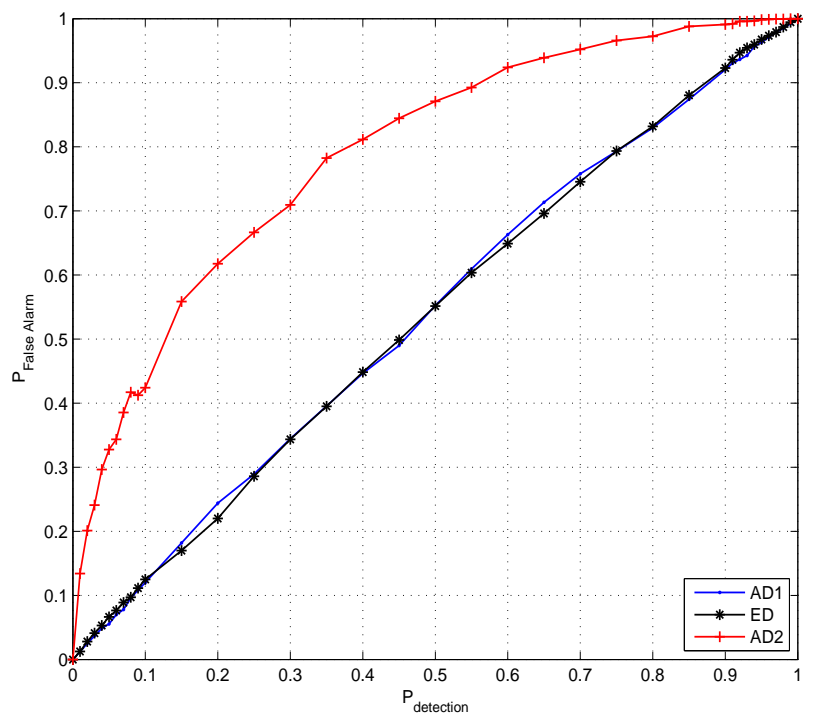

Fig. 3. ROC curves at $\mathrm{SNR}=-15 \mathrm{~dB}$

[4] H. Urkowitz, "Energy detection of unknown deterministic signals", Proceeding of the IEEE, Vol. 55, No. 4, pp. 523-531, Apr. 1967.

[5] B. Zayen, A. Hayar and D. Nussbaum, "Blind spectrum sensing for cognitive radio based on model selection", CrownCom 2008, 3rd International Conference on Cognitive Radio Oriented Wireless Networks and Communications, Mai 15-17 2008, Singapore.

[6] M. Haddad; A. Hayar; M, Debbah ;F, Hedi Mohamed, "Cognitive radio sensing information-theoretic criteria based", CrownCom 2007, 2nd International Conference on Cognitive Radio Oriented Wireless Networks and Communications, August 1-3, 2007, Orlando, USA.

[7] B. Zayen, A. Hayar and K.Kansnen, "'Blind Spectrum Sensing for Cognitive Radio Based on Signal Space Dimension Estimation", ICC'09, IEEE International Conference on Communications, June 14-18, 2009, Dresden, Germany.

[8] W. Guibene, M. Turki, A. Hayar and B. Zayen, "Development and Implementation of an Algebraic Detector for Spectrum Sensing", submitted to IEEE Journal on Selected Areas in Communications: Special Issue on Advances in Cognitive Radio Networking and Communications.

[9] Z. Tiganj and M. Mboup, "Spike detection and sorting: combining algebraic differentiation with ICA", "ICA", Paraty, Brazil, March 2009.

[10] Z. Tian and G. B. Giannakis, A wavelet approach to wideband spectrum sensing for cognitive radios, 1st International Conference on Cognitive Radio Oriented Wireless Networks and Communications p. 15, 2006. 